\title{
D-XY Critical Behavior in Cuprate Superconductors
}

\author{
T. Schneider and J. M. Singer \\ Physik-Institut, Universität Zürich, \\ Winterthurerstr. 190, CH-8057 Zürich, Switzerland
}

We outline the universal and finite temperature critical properties of the $3 D-X Y$ model, extended to anisotropic extreme type-II superconductors, as well as the universal quantum critical properties in $2 D$. On this basis we review: (i) the mounting evidence for $3 D-X Y$ behavior in optimally doped cuprate superconductors and the $3 D$ to $2 D$ crossover in the underdoped regime; (ii) the finite size limitations imposed by inhomogeneities; (iii) the experimental evidence for a $2 D-X Y$ quantum critical point in the underdoped limit, where the superconductor to insulator transition occurs; (iv) the emerging implications and constraints for microscopic models.

The starting point of the phenomenological theory of superconductivity is the GinzburgLandau Hamiltonian

$$
\begin{aligned}
\mathcal{H} & =\int d^{D} \mathbf{R}\left(\sum_{j=1}^{D} \frac{\hbar^{2}}{2 M_{j}}\left|\left(i \nabla_{j}+\frac{2 \pi}{\Phi_{0}} A_{j}(\mathbf{R})\right) \Psi\right|^{2}\right. \\
& \left.-r|\Psi|^{2}+\frac{u}{2}|\Psi|^{4}+\frac{|\operatorname{rot} \mathbf{A}|^{2}}{8 \pi}\right), j=(x, y, z) .
\end{aligned}
$$

$D$ is the dimensionality of the system, the complex scalar $\Psi(\mathbf{R})$ is the order parameter, $M$ the effective mass of the pair and $\mathbf{A}$ the vector potential. The pair carries a non-zero charge in addition to its mass. The charge $\left(\Phi_{0}=h c / 2 e\right)$ couples the order parameter to the electromagnetic field via the first term in $\mathcal{H}$.

If $\Psi$ and $\mathbf{A}$ are treated as classical fields, the relative probability $\mathcal{P}$ of finding a given configuration $\left[\Psi, \Psi^{*}, \mathbf{A}\right]$ is then

$$
\begin{aligned}
\mathcal{P}\left[\Psi, \Psi^{*}, \mathbf{A}\right] & =\exp \left(-\beta \mathcal{H}\left[\Psi, \Psi^{*}, \mathbf{A}\right]\right), \\
\beta & =1 /\left(k_{B} T\right) .
\end{aligned}
$$

The free energy $F$ follows from

$\exp \left(-\frac{F}{k_{B} T}\right)=Z=\int D[\mathbf{A}] D[\Psi] D\left[\Psi^{*}\right] \mathcal{P}\left[\Psi, \Psi^{*}, \mathbf{A}\right]$

where the partition function on the right hand side corresponds to an integral over all possible realizations of the vector potential $\mathbf{A}$, the order parameter $\Psi$ and its complex conjugate $\Psi^{*}$. Setting $e=0$, the free energy reduces to that for a normal to neutral superfluid transition, which is one of the best understood continuous phase transitions with unparalleled agreement between theory, simulations and experiment [1]. In extreme type-II superconductors, however, the coupling to vector potential fluctuations appears to be weak [2], but nonetheless these fluctuations drive the system - very close to criticality - to a charged critical point [3,4]. In any case, inhomogeneities prevent cuprate superconductors from entering this regime, due to the associated finite size effect. For these reasons, the neglect of vectorpotential fluctuations appears to be a reasonable starting point. In this case the vectorpotential in Hamiltonian Eq. (11) can be replaced by its most probable value. The critical properties at finite temperature are then those of the $3 D$ $X Y$ model, reminiscent to the lamda transition in superfluid helium, but extended to take the effective mass anisotropy into account [5, 6 .

The universal properties of the $3 D-X \bar{Y}$ universality class are characterized by a set of critical exponents, describing the asymptotic behavior of the correlation length $\xi_{i}^{ \pm}$, magnetic penetration depth $\lambda_{i}$, specific heat $A^{ \pm}$, etc., in terms of

$$
\xi_{i}^{ \pm}=\xi_{i, 0}^{ \pm}|t|^{-\nu}, \lambda_{i}=\lambda_{i, 0}|t|^{-\nu / 2}, C=\frac{A^{ \pm}}{\alpha}|t|^{-\alpha},
$$

where $3 \nu=2-\alpha$. As usual, in the above expression \pm refer to $t=T / T_{c}-1>0$ and $t<0$, respectively. The critical amplitudes $\xi_{i, 0}^{ \pm}, \lambda_{i, 0}^{2}, A^{ \pm}$, etc., are nonuniversal, but there are universal critical 


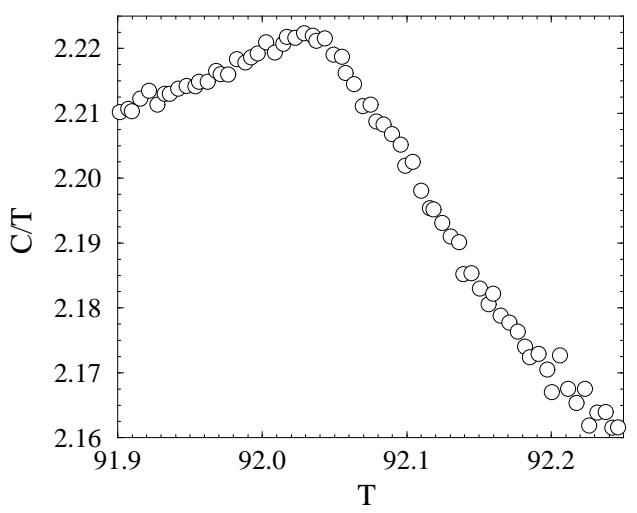

Figure 1. Specific heat coefficient $C / T$ $\left[m J /\left(g K^{2}\right)\right]$ versus $T[K]$ of $\mathrm{YBa}_{2} \mathrm{Cu}_{3} \mathrm{O}_{7-\delta}$ (sample YBCO3, [12]). The two arrows mark $T_{c} \approx$ $92.12 \mathrm{~K}$ and $T_{P} \approx 91.98 \mathrm{~K}$, respectively.

amplitude relations, including [6]

$$
\begin{aligned}
\left(k_{B} T_{c}\right)^{3} & =\left(\frac{\Phi_{0}^{2}}{16 \pi^{3}}\right)^{3} \frac{\xi_{x, 0}^{-} \xi_{y, 0}^{-} \xi_{z, 0}^{-}}{\lambda_{x, 0}^{2} \lambda_{y, 0}^{2} \lambda_{z, 0}^{2}} \\
& =\left(\frac{\Phi_{0}^{2}}{16 \pi^{3}}\right)^{3} \frac{\left(\mathcal{R}^{-}\right)^{3}}{A^{-} \lambda_{x, 0}^{2} \lambda_{y, 0}^{2} \lambda_{z, 0}^{2}} \\
\left(\mathcal{R}^{ \pm}\right)^{3} & =A^{ \pm} \xi_{x, 0}^{ \pm} \xi_{y, 0}^{ \pm} \xi_{z, 0}^{ \pm} .
\end{aligned}
$$

The singular part of the free energy density adopts in an applied magnetic field $H$ the scaling form [7]

$$
f_{s}=\frac{k_{B} T Q_{3}^{ \pm}}{\xi_{x}^{ \pm} \xi_{y}^{ \pm} \xi_{z}^{ \pm}} G_{3}^{ \pm}(\mathcal{Z}), \quad G_{3}^{ \pm}(0)=1,
$$

where

$$
\begin{aligned}
\mathbf{H} & =H(\cos (\phi) \sin (\delta), \sin (\phi) \sin (\delta), \cos (\delta)), \\
\mathcal{Z} & =\frac{1}{\Phi_{0}} \sqrt{H_{x}^{2} \xi_{y}^{2} \xi_{z}^{2}+H_{y}^{2} \xi_{x}^{2} \xi_{z}^{2}+H_{z}^{2} \xi_{x}^{2} \xi_{y}^{2}} .
\end{aligned}
$$

$\mathcal{R}^{ \pm}$and $Q_{3}^{ \pm}$are universal numbers, and $G_{3}^{ \pm}(\mathcal{Z})$ is an universal scaling function of its argument.

Provided that this scenario applies to cuprate superconductors, the implications include: (i) the universal relations hold irrespective of the dopant concentration and of the material; (ii) given the nonuniversal critical amplitudes of the correlation lengths, $\xi_{i, 0}^{ \pm}$, and the form of the universal scaling function $G_{3}^{ \pm}(\mathcal{Z})$, properties which can be derived from the free energy can be calculated close to the zero field transition. These properties include the

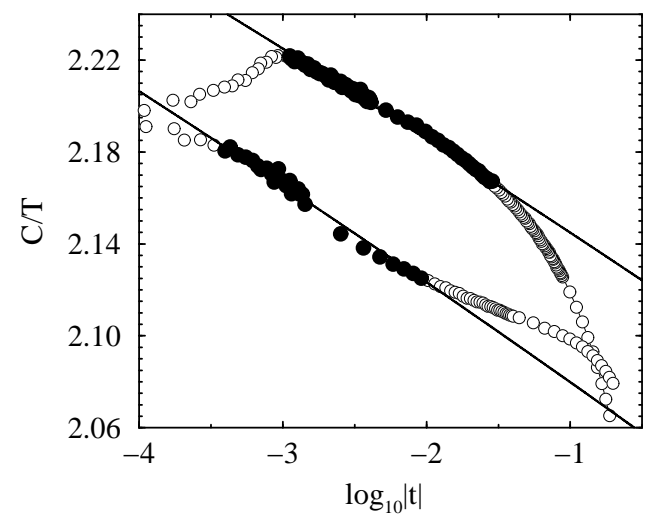

Figure 2. Specific heat coefficient $C / T$ $\left[m J /\left(g K^{2}\right)\right]$ versus $\log _{10}|t|$ for $\mathrm{YBa}_{2} \mathrm{Cu}_{3} \mathrm{O}_{7-\delta}$ (sample YBCO3, [12]) for $T_{c}=92.12 \mathrm{~K}$.

specific heat, magnetic torque, diamagnetic susceptibility, melting line, etc. It should be recognized that the universal relations, Eq. (4), also imply constraints on, e.g., isotope and pressure coefficients.

Although there is mounting evidence for $3 D$ $X Y$ universality in the cuprates [5 9,?, 11] it appears impossible to prove that unambiguously. Indeed, due to inhomogeneities, a solid always is homogeneous over a finite length $L$ only. In this case, the actual correlation length $\xi(t) \propto|t|^{-\nu}$ cannot grow beyond $L$ as $t \rightarrow 0$, and the transition appears rounded. Due to this finite size effect, the specific heat peak occurs at a temperature $T_{P}$ shifted from the homogeneous system by an amount $L^{-1 / \nu}$, and the magnitude of the peak located at temperature $T_{P}$ scales as $L^{\alpha / \nu}$. To quantify this point we show in Fig. 1 the measured heat coefficient of $\mathrm{YBa}_{2} \mathrm{Cu}_{3} \mathrm{O}_{7-\delta}$ 121 around the peak. The rounding and the shape of the specific heat coefficient clearly exhibit the characteristic behavior of a system in confined dimensions, i.e., rod or cube shaped inhomogeneities [13]. A finite size scaling analysis 114. reveals inhomogeneities with a characteristic length scale ranging from 300 to $400 \AA$, in the $\mathrm{YBa}_{2} \mathrm{Cu}_{3} \mathrm{O}_{7-\delta}$ samples $\mathrm{YBCO} 3, \mathrm{UBC} 2$ and UBC1 of Ref. 12]. For this reason, deviations from $3 D-X Y$ critical behavior around $T_{P}$ do not 


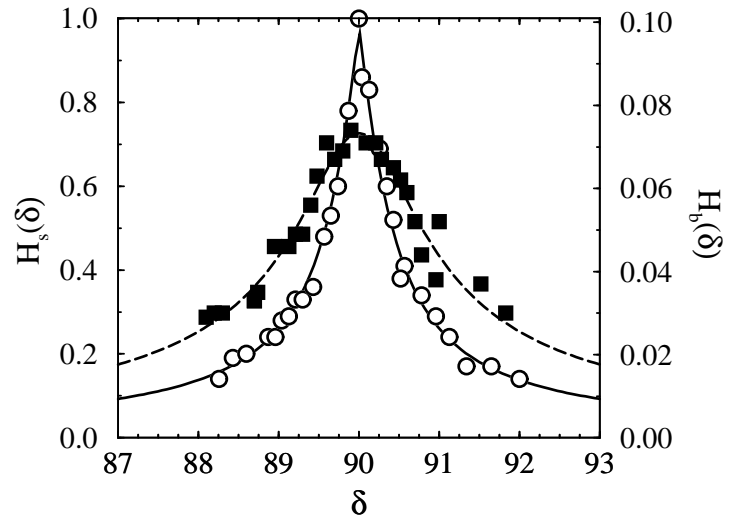

Figure 3. $H^{*}(\delta)$ for $\mathrm{Bi}_{2} \mathrm{Sr}_{2} \mathrm{CaCu}_{2} \mathrm{O}_{8+\delta}$ with $T_{c}=$ $84.1 K$ for $T=79.5 K$ (o) and $T=82.8 K(\boldsymbol{\square})$. Experimental data are taken from [16], the solid lines are derived from the respective $2 D\left(H_{s}\right.$, 'slab', - $)$ and $3 D\left(H_{b}\right.$, 'bulk' with $\gamma=77$, -) scaling forms.

signal the failure of $3 D-X Y$ universality, as previously claimed 12, but reflect a mere finite size effect at work. Indeed, from Fig. 2 it is seen that the finite size effect makes it impossible to enter the asymptotic critical regime. To set the scale we note that in the $\lambda$-transition of ${ }^{4} \mathrm{He}$ the critical properties can be probed down to $|t|=10^{-9}$ 1.15. In Fig. 2 we marked the intermediate regime where consistency with $3 D-X Y$ critical behavior, i.e., with $C / T=\widetilde{A}^{ \pm} 10^{-\alpha \log _{10}|t|}+\widetilde{B}^{ \pm}$ for $\alpha=-0.013$ and $\widetilde{A}^{+} / \widetilde{A}^{-}=1.07$, can be observed. The upper branch corresponds to $T<T_{c}$ and the lower one to $T>T_{c}$. The open circles closer to $T_{c}$ correspond to the finite size affected region, while further away the temperature dependence of the background, usually attributed to phonons, becomes significant. Hence, due to the finite size effect and the temperature dependence of the background the intermediate regime is bounded by the temperature region where the data depicted in Fig. 2 fall nearly on straight lines. To provide quantitative evidence for $3 D$ $X Y$ universality in this regime, we invoke the universal relation (4) and calculate $T_{c}$ from the critical amplitudes of specific heat and penetra-

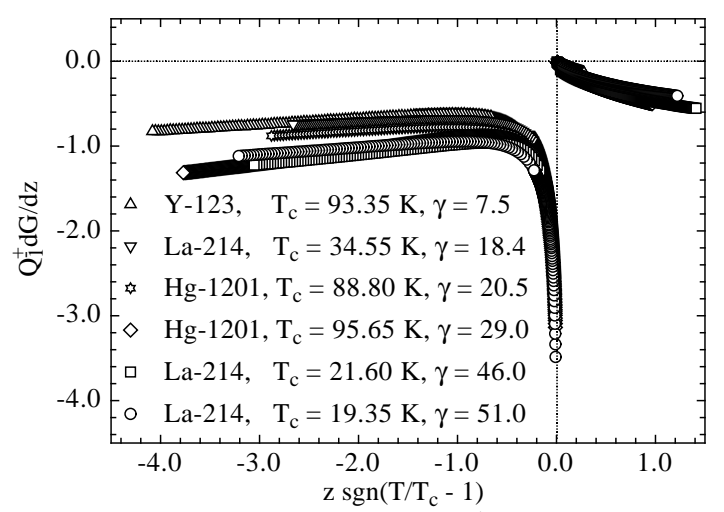

Figure 4. Scaling function $d G_{3}^{ \pm}(\mathcal{Z}) / d \mathcal{Z}$ derived from the angular dependence of the magnetic torque for $\mathrm{YBa}_{2} \mathrm{Cu}_{3} \mathrm{O}_{6.93}, \mathrm{La}_{1.854} \mathrm{Sr}_{0.146} \mathrm{CuO}_{4}$, $\mathrm{HgBa}_{2} \mathrm{CuO}_{4.108}, \mathrm{HgBa}_{2} \mathrm{CuO}_{4.096}$,

$\mathrm{La}_{1.914} \mathrm{Sr}_{0.086} \mathrm{CuO}_{4}$ and $\mathrm{La}_{1.920} \mathrm{Sr}_{0.080} \mathrm{CuO}_{4}$.

tion depth. Using $A^{+}=8.4 \cdot 10^{20} \mathrm{~cm}^{-3}$, derived from the data shown in Fig. 2 for sample YBCO3 with $T_{c}=92.12 \mathrm{~K}, \lambda_{a, 0}=1153 \AA$ A,$\lambda_{b, 0}=968 \AA$ and $\lambda_{c, 0}=8705 \AA$, derived from magnetic torque measurements on a sample with $T_{c}=91.7 \mathrm{~K}$ [7], as well as the universal numbers $A^{+} / A^{-}=1.07$ and $\mathcal{R}^{-} \approx 0.59$, we obtain $T_{c}=88.2 K$. Hence, the universal $3 D-X Y$ relation (1) is remarkably well satisfied.

Another difficulty results from the pronounced anisotropy of the cuprates: a convenient measure is the effective mass parameter $\gamma=\sqrt{M_{\|} / M_{\perp}}$, which depends on the dopant concentration. Even though the strength of thermal fluctuations grows with increasing $\gamma$, they are slightly away from $T_{c}$ essentially two-dimensional. Accordingly, the intermediate $3 D-X Y$ critical regime shrinks, and the corrections to scaling are expected to become significant. An experimental demonstration of the temperature driven dimensional crossover is shown in Fig. 3 in terms of the angular dependence of the onset field $H^{*}$, where a measurable resistance is observed [16]. $H^{*}(\delta)$ follows from $\mathcal{Z}\left(H^{*}\right)=\mathcal{Z}^{*}$. For superconducting sheets of thickness $d_{s}$, corresponding to $2 D$, the argument of the scaling function $G_{2}^{ \pm}(\mathcal{Z})$ is given by 


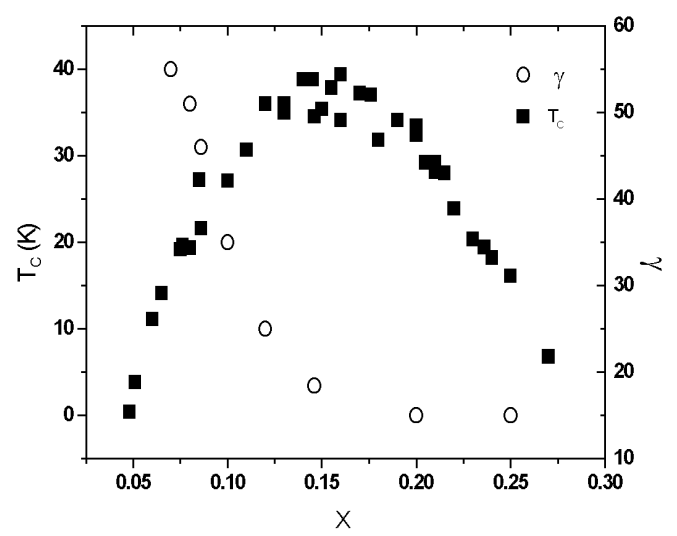

Figure 5. $T_{c}$ and $\gamma=\sqrt{M_{\perp} / M_{\|}}$versus dopant concentration $x$ for $\mathrm{La}_{2-\mathrm{x}} \mathrm{Sr}_{\mathrm{x}} \mathrm{CuO}_{4}$.

17, 18.

$$
\mathcal{Z}=\left(\frac{H}{\Phi_{0}}\left(\xi_{\|}^{ \pm}\right)^{2}|\cos (\delta)|+\frac{H^{2}}{\Phi_{0}^{2}}\left(\xi_{\|}^{ \pm}\right)^{2} d_{s}^{2} \sin ^{2}(\delta)\right)^{1 / 2}
$$

Noting that Eqs. (6) and (7) lead to distinct bell-shaped $(3 D)$ and cusp-like $(2 D)$ behavior around $\delta=90^{\circ}$, respectively, these measurements clearly illustrate the temperature driven dimensional crossover. Indead, as seen in Fig. 3, at $T=79.5 K H^{*}(\delta)$ mirrors a $2 D$ film behavior, while closer to $T_{c}$ at $T=82.8 \mathrm{~K} 3 \mathrm{D}$ bulk behavior appears. To illustrate the difficulties associated with this crossover we show in Fig. 1 estimates for the derivative of the universal scaling function $G_{3}^{ \pm}(\mathcal{Z})$ derived from magnetic torque measurements [11]. Even though the qualitative behavior is the same for all samples, the deviations increase systematically with increasing $\gamma$. This systematics cannot be attributed to the experimental uncertainties of about $40 \%$. It is more likely that it reflects the $3 D$ to $2 D$ crossover and the associated reduction of the $3 D-X Y$ fluctuation dominated regime, requiring corrections to scaling. Indeed, in the derivation of the scaling function from the experimental data, both, corrections to scaling and finite size effects have not been considered.

In materials, such as $\mathrm{La}_{2-x} \mathrm{Sr}_{\mathrm{x}} \mathrm{CuO}_{4}$, where the underdoped regime is experimentally accessible,

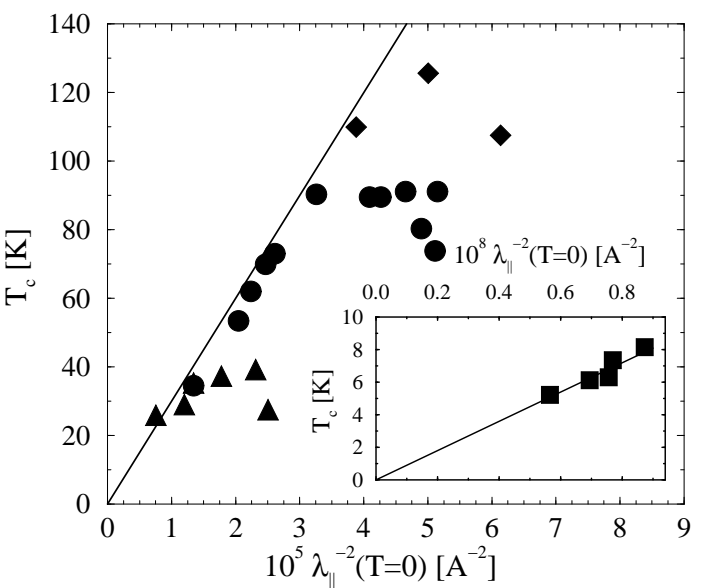

Figure 6. $T_{c}$ versus $\lambda_{\|}^{-2}(T \rightarrow 0)$ as obtained from $\mu \mathrm{SR}$ measurements for $\mathrm{La}_{2-\mathrm{x}} \mathrm{Sr}_{\mathrm{x}} \mathrm{CuO}_{4}(\mathbf{\Lambda})$, $\mathrm{YBa}_{2} \mathrm{Cu}_{3} \mathrm{O}_{7-\mathrm{x}}, \mathrm{Bi}_{2} \mathrm{Sr}_{2} \mathrm{Ca}_{1-\mathrm{x}} \mathrm{Y}_{\mathrm{x}} \mathrm{Cu}_{2} \mathrm{O}_{8+\delta}(\bullet)$, and $\mathrm{Tl}_{2} \mathrm{Ba}_{2} \mathrm{Ca}_{2} \mathrm{Cu}_{3} \mathrm{O}_{10}(\bullet)$. Data taken from [23]. The straight line marks $T_{c}=3 \cdot 10^{8} \lambda_{\|}^{-2}$ with $T_{c}$ in $[K]$ and $\lambda_{\|}$in $[\AA]$. Inset: $T_{c}$ vs. $\lambda_{\|}^{-2}(T=0)$ of $\mathrm{Bi}_{2+\mathrm{x}} \mathrm{Sr}_{2-\mathrm{x}} \mathrm{CuO}_{6+\delta}(\mathbf{\square})$; the straight line is a guide to the eye. Data taken from 24].

a strict $3 D$ - to $2 D-X Y$ crossover occurs. By approaching the underdoped limit $x=x_{u} \approx 0.05$, $\gamma=\sqrt{M_{\perp} / M_{\|}}$becomes very large (see Fig. 5) and $T_{c}$ vanishes. Here the materials correspond to a stack of independent sheets of thickness $d_{s}$ As there is a phase transition line with an endpoint $T_{c}\left(x=x_{u}\right)=0$, one expects a doping driven $2 D-X Y$ insulator to superconductor transition at $T=0$. For such a transition the scaling theory of quantum critical phenomena predicts 19 21]

$$
\begin{array}{r}
\lim _{\delta \rightarrow 0} \frac{1}{d_{s}^{2}}\left(T_{c}(\delta)\right)^{2} \lambda_{x}^{2}(\delta, T=0) \lambda_{y}^{2}(\delta, T=0)= \\
=\frac{1}{Q_{2,0}^{2}}\left(\frac{\Phi_{0}^{2}}{16 \pi^{3} k_{B}}\right)^{2}
\end{array}
$$

to be universal. $\delta=\left(x-x_{u}\right) / x_{u}$ is the control parameter, $Q_{2,0}$ is an universal number and

$$
T_{c} \propto \delta^{z \bar{\nu}}, \quad \lambda_{i}^{2}(\delta, T=0) \propto \delta^{-\bar{\nu}} .
$$

$z$ is the dynamic critical exponent and $\bar{\nu}$ the exponent of the correlation length. In Fig. 6 we depict experimental data in terms of $T_{c}$ versus 
$1 / \lambda_{\|}^{2}(T=0)$. As $T_{c}$ approaches the underdoped limit, the data appear to merge on the solid line. In this context it should be emphasized that the data, with the exception of $\mathrm{Bi}_{2+x} \mathrm{Sr}_{2-x} \mathrm{CuO}_{6+\delta}$, are rather far away from the asymptotic regime where Eq. (8) is expected to apply. Moreover, $d_{s}$ is known to adopt material dependent values 22. Nevertheless, the data collected in Figs. 5 and 6 clearly point to a quantum phase transition in $D=2$ at $x=x_{u}$ where $T_{c}$ vanishes and $\lambda_{\|}^{2}(T=0)$ tends to infinity. Hence, there is strong evidence for a $2 D-X Y$ quantum phase transition, where Eq. (8) applies and $d T_{c} / d\left(1 / \lambda_{\|}^{2}(T=0)\right)$ is not universal, as suggested by Uemura et al [23], but depends on $d_{s}$.

To summarize, there is mounting evidence for intermediate $D-X Y$ critical behavior, a $3 D$ - to $2 D$ - crossover as the underdoped limit is approached and for the occurrence of a quantum superconductor to insulator transition at the underdoped limit in $D=2$. Emerging implications and constraints for microscopic models include: (i) in the experimentally accessible temperature regime and close to optimum doping, there is remarkable consistency with $3 D-X Y$ universality; (ii) close to criticality the symmetry of the order parameter is either d-wave or s-wave; (iii) the decrease of $T_{c}$ in the underdoped regime mirrors the dimensional crossover, enhancing thermal fluctuations and the competition with quantum fluctuations which suppress superconductivity at the underdoped limit; (iv) the enhanced thermal and quantum fluctuations reduce the single particle density of states at the chemical potential. This reduction leads to a pseudogap above $T_{c}$; (v) these fluctuations imply the existence of phase uncorrelated pairs above $T_{c}$ and invalidate mean-field treatments, including the Fermi liquid approach in the normal state; etc.

For a more elaborate review of the $D-X Y$ behavior in cuprate superconductors we refer to Ref. 25.

The authors are grateful to J. Hofer for very useful comments and suggestions on the subject matter.

\section{REFERENCES}

1. A. Singsaas and G. Ahlers, Phys. Rev. B30 (1984) 5103.

2. D. S. Fisher et al, Phys. Rev. B43 (1991) 130.

3. I. F. Herbut and Z. Tesanovic, Phys. Rev. Lett. 76 (1996) 4588.

4. A. K. Nguyen and A. Sudbo, condmat/9907385.

5. T. Schneider and D. Ariosa, Z. Phys. B89 (1992) 249.

6. T. Schneider and H. Keller, Int. J. Mod. Phys. B8 (1993) 487.

7. T. Schneider et al, Eur. Phys. J. B3 (1998) 413.

8. M. A. Hubbard et al, Physica C259 (1996) 309.

9. S. Kamal et al, Phys. Rev. Lett. 73 (1994) 1845; Phys. Rev. B58 (1998) R8933.

10. V. Pasler et al, Phys. Rev. Lett. 81 (1998) 1094.

11. J. Hofer et al, Phys. Rev. B60 (1999) 1332; Preprint, 1999.

12. M. Charalambous et al, Phys. Rev. Lett. 83 (1999) 2042.

13. E. Schultka and E. Manusakis, Phys. Rev. Lett. 75 (1995) 2710; cond-mat/9602085; cond-mat/9702216; cond-mat/9811251.

14. T. Schneider and J. M. Singer, condmat/9911352.

15. S. Metha et al, J. Low Temp. Phys. 114 (1999) 467.

16. E. Silva et al., Physica C214 (1993) 175.

17. T. Schneider and A. Schmidt, Phys. Rev. B47 (1993) 5915.

18. T. Schneider and J. M. Singer, condmat/9911411.

19. Min-Chui Cha et al, Phys. Rev. B44 (1991) 6883.

20. K. Kim and P. B. Weichman, Phys. Rev. B43 (1991) 13583.

21. T. Schneider, Acta Physica Polonica A91 (1997) 203.

22. T. Schneider and J. M. Singer, Physica C313 (1999) 188.

23. Y. J. Uemura et al, Phys. Rev. Lett. 59 (1987) 1045; Phys. Rev. B38 (1988) 909.

24. E. Janod. PhD thesis, CEA Grenoble, 1996.

25. T. Schneider and J. M. Singer, Phase Transition Approach To High Temperature Super- 
conductivity, Imperial College Press, London, 2000. 